\title{
A potential prognostic IncRNA signature for predicting survival in patients with bladder urothelial carcinoma
}

\author{
Zhenyu Bao ${ }^{1, *}$, Weitao Zhang ${ }^{2, *}$, Dong Dong ${ }^{1}$ \\ ${ }^{1}$ Shanghai Key Laboratory of Regulatory Biology, Institute of Biomedical Sciences, School of Life Sciences, East China Normal \\ University, Shanghai, China \\ ${ }^{2}$ Urology Surgery Department, Affiliated Hospital of Taishan Medical University, Shandong, China \\ *These authors have contributed equally to this work \\ Correspondence to: Dong Dong, email: ddong.ecnu@gmail.com \\ Keywords: long non-coding RNA, prognosis, bladder urothelial carcinoma \\ Received: October 15, $2016 \quad$ Accepted: December 08, $2016 \quad$ Published: January 02, 2017
}

\section{ABSTRACT}

Increasing evidence has highlighted the critical roles of long non-coding RNA (IncRNA) in cancer development and progression. However, the prognostic power of expression-based IncRNA signature for predicting overall survival in patients with Bladder Urothelial Carcinoma (BLCA) has not been investigated. Here, we performed a comprehensive analysis for IncRNA expression profiles and corresponding clinical information of 234 BLCA patients from The Cancer Genome Atlas (TCGA). We established a set of four-IncRNAs that were significantly associated with BLCA patients' survival. Using the prognostic four-IncRNA signature, we successfully classified the BLCA patients into high-risk and low-risk groups, and the prognostic power of the four-IncRNA signature was further validated in the testing dataset and entire dataset. Multivariate Cox regression and stratified analyses demonstrated that the prognostic power of the four-IncRNA signature was independent of other clinical variables. Functional enrichment analyses suggested the four prognostic IncRNAs may be involved in known BLCA-related biological processes and pathways. Our results demonstrated that the four-IncRNA signature could be novel independent biomarkers for predicting survival in patients with BLCA.

\section{INTRODUCTION}

Bladder cancer is the ninth most common malignancy worldwide [1]. It has been estimated about 80000 newly diagnosed bladder cancer cases in the United States in 2015 [2]. Bladder Urothelial Carcinoma (BLCA) is the most common histological subtype of bladder cancer. Overall, about $70 \%$ of bladder tumors are non-muscleinvasive bladder cancer, and the others are muscle-invasive bladder cancer [3]. Despite recent advances in the surgical technique, the overall survival of BLCA patients has not been dramatically improved, and the five-year survival rate remains at only $50-60 \%$ [4-6]. Therefore, it is necessary to identify novel independent biomarkers for diagnostic and prognosis and to develop new targeted therapies for BLCA patients.

Long non-coding RNAs (lncRNAs) are an important category of non-coding RNAs (ncRNAs) with little or no protein-coding capacity, which range from 200 nucleotides to multiple kilobases in length $[7,8]$. Accumulated evidence suggests that IncRNAs play crucial roles in regulating gene expression at transcriptional, posttranscriptional and epigenetic levels $[7,9]$, and participate in various biological processes and pathways, such as transcriptional regulation, cell growth and tumorigenesis $[10,11]$. Like mRNA and miRNA, some well-studied lncRNAs have been found to play critical oncogenic or tumor suppressive roles in various types of cancers [12-14]. For instance, HOTAIR, MALAT1 and $C R N D E$ have been showed as oncogenic roles [1525], while GAS5, MEG3 and lincRNA-p21 as tumor suppressive roles [26-32]. Currently, several expressionbased IncRNA signatures have been identified in glioblastoma [33], oesophageal squamous cell carcinoma [34], breast cancer [35], colorectal cancer [36], non-small cell lung cancer [37], multiple myeloma [38] and ovarian cancer [39], highlighting their potential roles as novel independent biomarkers for cancer prognosis. For bladder 
cancer, recent studies have also revealed that lncRNAs (HOTAIR, SPRY4-IT1, SUMO1P3 and PANDAR) are aberrantly expressed in BLCA patients [40-43]. However, the prognostic power of expression-based lncRNA signature for predicting BLCA patients' survival remains unclear.

In this work, we performed a comprehensive analysis for lncRNA expression profiles and corresponding clinical information of BLCA patients in the TCGA training dataset. We identified four lncRNAs significantly associated with patients' survival and constructed a fourlncRNA signature that can effectively predict patients' survival. The testing dataset and entire dataset further validated the prognostic power of the four-lncRNA signature. Our results demonstrated the four-lncRNA signature can function as novel independent biomarkers for BLCA prognosis and provide novel insights into understanding of the underlying molecular mechanism of BLCA.

\section{RESULTS}

\section{Identification of prognostic IncRNAs associated with patients' survival from the training dataset}

The 234 patients with BLCA were randomly divided into a training dataset $(n=117)$ and a testing dataset $(n=117)$. The training dataset was analyzed to identify prognostic lncRNAs. At first, we performed a univariate Cox regression analysis to evaluate the association between the expression profiles of each lncRNA and patients' survival in the training dataset. The result showed that four lncRNAs were identified as prognostic lncRNAs ( $p$-value $<0.001)$. The detailed information of these four IncRNAs was showed in Table 1. Among these prognostic lncRNAs, the lncRNAs (AC005682.5 and CTD-2231H16.1) with higher expression profiles were associated with shorter survival (coefficient $>0$ ), while the remaining two lncRNAs (CTB-92J24.2 and RP11-727F15.13) with higher expression profiles were associated with longer survival (coefficient $<0)$.

\section{Construction and validation of a four-IncRNA signature for predicting patients' survival in the training dataset}

To construct a prognostic signature, these four lncRNAs were analyzed using a multivariate Cox regression analysis in the training dataset with survival as the dependent variable and other clinical information as covariables. Then we constructed a prognostic signature by integrating the expression profiles of the four lncRNAs and corresponding estimated regression coefficient derived from above multivariate Cox regression analysis as follows: Risk score $=(0.371 \times$ expression value of
$A C 005682.5)+(0.175 \times$ expression value of $C T D-$ $2231 H 16.1)+(-0.251 \times$ expression value of CTB-92J24.2) $+(-0.232 \times$ expression value of RP11-727F15.13). With the four-lncRNA signature, we calculated a risk score for each patient in the training dataset and ranked them according to increased risk score. Thus, 117 patients of the training dataset were classified into a high-risk group $(n=59)$ and a low-risk group $(n=58)$ using the median risk score (-1.12) as the cutoff point. The Kaplan-Meier analysis showed a significant difference in patients' survival between the high-risk group and the low-risk group (log-rank test $p$-value $=8.94 \mathrm{E}-09$; Figure 1A). Patients in the high-risk group had significantly shorter survival (median 5.60 months) than those in the low-risk group (median 7.52 months). To evaluate how well the four-lncRNA signature for predicting the 5-year survival, the time-dependent ROC curve analysis was carried out. The AUC for the four-lncRNA signature was 0.807 at the survival of five years (Figure 1B), demonstrating the competitive performance of the four-lncRNA signature for survival prediction in the training dataset. In the univariate Cox regression analysis of the training dataset, the fourlncRNA risk score were significantly associated with patients' survival ( $p$-value $=3.33 \mathrm{E}-05, \mathrm{HR}=23.141,95 \%$ $\mathrm{CI}=$ 5.248-102.043; Table 2).

The distribution of the risk score, overall survival and prognostic lncRNA expression profiles in 117 patients of the training dataset were showed in Figure 1C, ranked according to increased risk score. Patients with high-risk scores had higher mortality than patients with low-risk scores. For patients with high risk scores, the expression profiles of lncRNAs (AC005682.5 and CTD-2231H16.1) are significantly up-regulated, while the remaining two lncRNAs (CTB-92J24.2 and RP11-727F15.13) were down-regulated.

\section{Validation of the four-IncRNA signature for survival prediction in the testing dataset and entire dataset}

To validate the prognostic power of the fourlncRNA signature for survival prediction, 117 patients of the testing dataset were divided into a high-risk group $(n=62)$ and a low-risk group $(n=55)$ with the same lncRNA signature and cutoff point derived from the training dataset. In consistent with the findings in the training dataset, the result showed that a significantly different survival between the high-risk group and the low-risk group (log-rank test $p$-value $=3.49 \mathrm{E}-02$, median 6.62 months vs. 6.97 months; Figure 2A). The AUC for the four-lncRNA signature was 0.656 at the survival of five years in the testing dataset. In the univariate Cox regression analysis of the testing dataset, the fourlncRNA risk score were significantly associated with patients' survival ( $p$-value $=3.97 \mathrm{E}-02, \mathrm{HR}=2.365,95 \%$ $\mathrm{CI}=1.042-5.372$; Table 2). 
Table 1: The detailed information of four prognostic IncRNAs significantly associated with overall survival in patients with BLCA

\begin{tabular}{|c|c|c|c|c|c|}
\hline Ensembl ID & Gene symbol & Chromosomal position & $P$ value ${ }^{a}$ & $\begin{array}{c}\text { Hazard } \\
\text { ratio }^{\mathrm{a}}\end{array}$ & Coefficient $\mathbf{t}^{\mathrm{t}}$ \\
\hline ENSG00000228649 & AC005682.5 & $\operatorname{chr} 7: 22,854,178-22,861,579(+)$ & $3.39 \mathrm{E}-04$ & 1.613 & 0.371 \\
\hline ENSG00000249430 & CTD-2231H16.1 & chr5: 92,151-139,863(+) & 8.44E-04 & 0.175 & 0.175 \\
\hline ENSG00000269397 & $C T B-92 J 24.2$ & chr19: $23,927,788-23,929,287(+)$ & $2.22 \mathrm{E}-04$ & 0.609 & -0.251 \\
\hline ENSG00000269463 & $R P 11-727 F 15.13$ & chr11: $62,807,682-62,808,063(-)$ & $6.88 \mathrm{E}-04$ & 0.736 & -0.232 \\
\hline
\end{tabular}

${ }^{\mathrm{a}, \mathrm{b}}$ Derived from the univariate and multivariate Cox regression analyses in 117 patients of the training dataset.
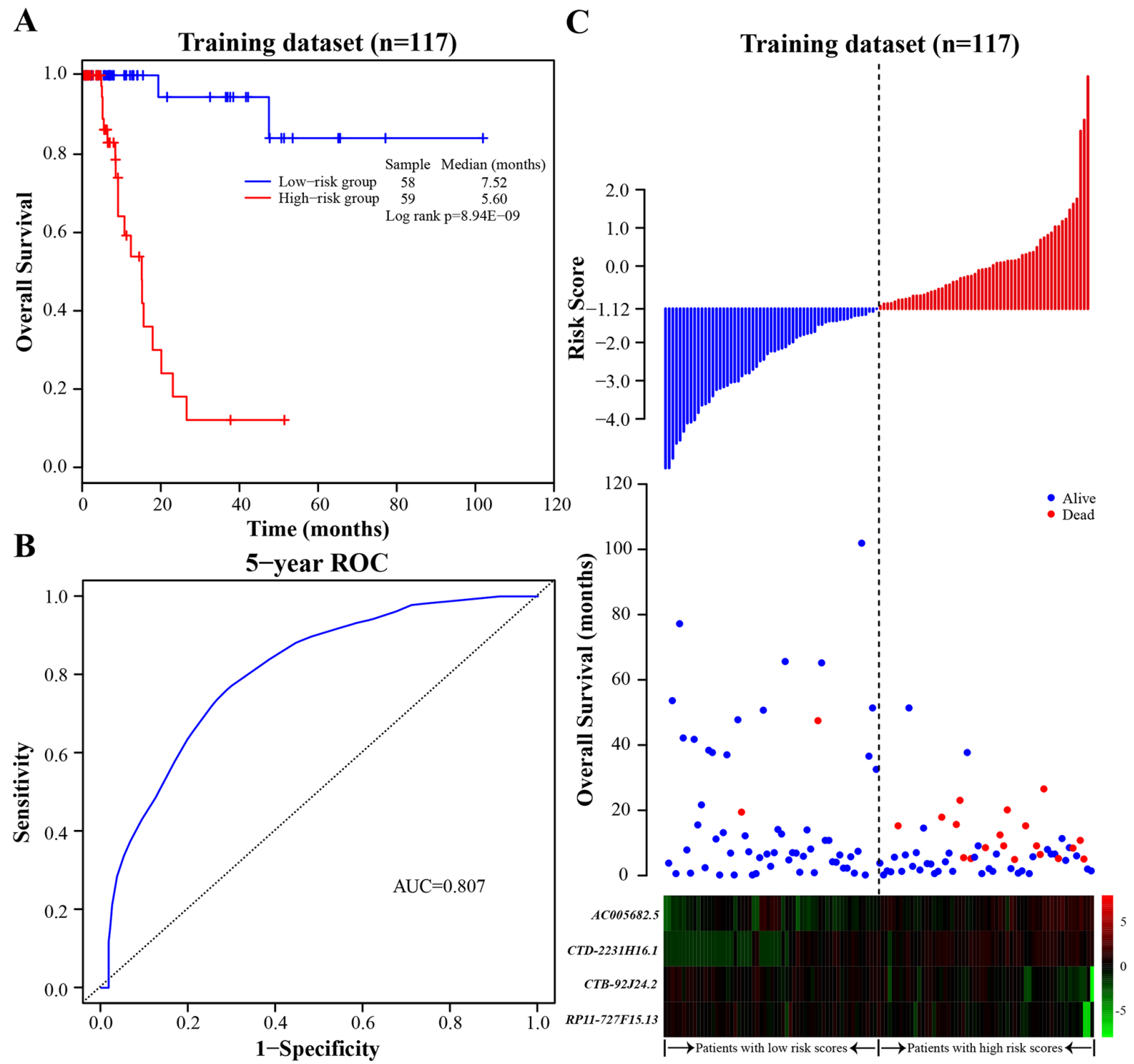

Figure 1: The four-IncRNA signature in prognosis of overall survival of BLCA patients in the training dataset. A. The Kaplan-Meier curves of overall survival between high-risk and low-risk patients in the training dataset. B. The ROC curve for survival prediction by the four-IncRNA signature within five years as the defining point in the training dataset. C. The four-lncRNA risk score distribution, overall survival of patients and heatmap of the four-lncRNA expression profiles in the training dataset. 
Table 2: Univariate and multivariate Cox regression analyses in each dataset

\begin{tabular}{|c|c|c|c|c|c|c|}
\hline \multirow{2}{*}{ Variables } & \multicolumn{3}{|c|}{ Univariate analysis } & \multicolumn{3}{|c|}{ Multivariate analysis } \\
\hline & HR & $95 \%$ CI of HR & $P$ value & HR & $95 \%$ CI of HR & $P$ value \\
\hline \multicolumn{7}{|l|}{ Training dataset $(n=117)$} \\
\hline \multicolumn{7}{|l|}{ Four-lncRNA risk score } \\
\hline Low risk/High risk & 23.141 & $5.248-102.043$ & 3.33E-05 & 21.761 & $4.637-102.129$ & $9.44 \mathrm{E}-05$ \\
\hline \multicolumn{7}{|l|}{ Age } \\
\hline$\leq 65 />65$ & 1.072 & $0.444-2.591$ & 0.876 & 0.700 & $0.237-2.071$ & 0.520 \\
\hline \multicolumn{7}{|l|}{ Gender } \\
\hline Female/Male & 0.786 & $0.304-2.031$ & 0.620 & 0.909 & $0.331-2.498$ & 0.853 \\
\hline \multicolumn{7}{|l|}{ Subtype } \\
\hline Non-Papillary/Papillary & 0.343 & $0.100-1.173$ & $8.82 \mathrm{E}-02$ & 0.290 & $0.060-1.401$ & 0.123 \\
\hline \multicolumn{7}{|l|}{ Stage } \\
\hline II & 1 (reference) & & & 1 (reference) & & \\
\hline III & 2.408 & $0.598-9.701$ & 0.217 & 0.246 & $0.031-1.946$ & 0.184 \\
\hline IV & 3.350 & $0.944-11.882$ & $6.13 \mathrm{E}-02$ & 1.369 & $0.335-5.591$ & 0.662 \\
\hline \multicolumn{7}{|l|}{ Testing dataset $(n=117)$} \\
\hline \multicolumn{7}{|l|}{ Four-lncRNA risk score } \\
\hline Low risk/High risk & 2.365 & $1.042-5.372$ & $3.97 \mathrm{E}-02$ & 2.459 & $1.067-5.666$ & $3.46 \mathrm{E}-02$ \\
\hline \multicolumn{7}{|l|}{ Age } \\
\hline$\leq 65 />65$ & 1.148 & $0.479-2.754$ & 0.756 & 1.333 & $0.549-3.240$ & 0.525 \\
\hline \multicolumn{7}{|l|}{ Gender } \\
\hline Female/Male & 1.199 & $0.481-2.991$ & 0.697 & 1.229 & $0.488-3.096$ & 0.662 \\
\hline \multicolumn{7}{|l|}{ Subtype } \\
\hline Non-Papillary/Papillary & 0.507 & $0.175-1.473$ & 0.212 & 0.633 & $0.213-1.885$ & 0.411 \\
\hline \multicolumn{7}{|l|}{ Stage } \\
\hline II & 1 (reference) & & & 1 (reference) & & \\
\hline III & 1.680 & $0.515-5.484$ & 0.390 & 1.479 & $0.422-5.180$ & 0.541 \\
\hline IV & 2.472 & $0.791-7.720$ & 0.119 & 2.024 & $0.634-6.468$ & 0.234 \\
\hline \multicolumn{7}{|l|}{ Entire dataset $(n=234)$} \\
\hline \multicolumn{7}{|l|}{ Four-lncRNA risk score } \\
\hline Low risk/High risk & 5.581 & $2.839-10.972$ & $6.18 \mathrm{E}-07$ & 4.975 & $2.527-9.795$ & $3.45 \mathrm{E}-06$ \\
\hline \multicolumn{7}{|l|}{ Age } \\
\hline$\leq 65 />65$ & 1.118 & $0.603-2.073$ & 0.722 & 1.202 & $0.643-2.248$ & 0.564 \\
\hline \multicolumn{7}{|l|}{ Gender } \\
\hline Female/Male & 0.994 & $0.516-1.915$ & 0.985 & 1.145 & $0.587-2.233$ & 0.691 \\
\hline \multicolumn{7}{|l|}{ Subtype } \\
\hline Non-Papillary/Papillary & 0.433 & $0.194-0.967$ & 4.12E-02 & 0.553 & $0.242-1.268$ & 0.162 \\
\hline
\end{tabular}




\begin{tabular}{|c|c|c|c|c|c|c|}
\hline \multirow{2}{*}{ Variables } & \multicolumn{3}{|c|}{ Univariate analysis } & \multicolumn{3}{|c|}{ Multivariate analysis } \\
\hline & HR & $95 \%$ CI of HR & $P$ value & HR & $95 \%$ CI of HR & $P$ value \\
\hline \multicolumn{7}{|l|}{ Stage } \\
\hline II & 1 (reference) & & & 1 (reference) & & \\
\hline III & 2.070 & $0.840-5.099$ & 0.114 & 1.070 & $0.383-2.992$ & 0.898 \\
\hline IV & 2.917 & $1.256-6.775$ & $1.28 \mathrm{E}-02$ & 1.866 & $0.774-4.497$ & 0.165 \\
\hline
\end{tabular}

When the four-lncRNA signature was further applied to the entire TCGA dataset, similar results were observed. As in the training and testing dataset, the fourlncRNA signature could also classify 234 patients of the entire dataset into a high-risk group $(n=121)$ and a lowrisk group $(n=113)$ with significantly different survival (log-rank test $p$-value $=3.89 \mathrm{E}-08$, median 6.43 months $v s$. 7.33 months; Figure 2B). In the entire dataset, the AUC for the four-lncRNA signature was 0.758 at the survival of five years. The univariate Cox regression analysis also demonstrated that the four-lncRNA risk score was significantly associated with patients' survival in the entire dataset $(p$-value $=6.18 \mathrm{E}-07, \mathrm{HR}=5.58,95 \% \mathrm{CI}$ $=2.84-10.97$; Table 2). Taken together, the above results demonstrated good reliability and reproducibility of the four-lncRNA signature for predicting BLCA patients' survival.

\section{Independence of the four-IncRNA signature for survival prediction from other clinical variables}

To evaluate whether the prognostic power of the four-lncRNA signature was independent of other clinical variables including age, gender, subtype and tumor stage, the multivariate Cox regression analyses were first carried out in each dataset. The results from the three datasets demonstrated that the four-lncRNA risk score was significantly associated with patients' survival. Specifically, the four-lncRNA signature still maintained an independent association with survival after adjustment for other clinical variables in the training dataset ( $p$-value $=9.44 \mathrm{E}-05, \mathrm{HR}=21.761,95 \% \mathrm{CI}=4.637-102.129)$, testing dataset $(p$-value $=3.46 \mathrm{E}-02, \mathrm{HR}=2.459,95 \%$ $\mathrm{CI}=1.067-5.666)$ and entire dataset $(p$-value $=3.45 \mathrm{E}$ $06, \mathrm{HR}=4.975,95 \% \mathrm{CI}=2.527-9.795$; Table 2). Next, stratified analyses were then performed according to age, tumor stage and subtype, respectively. First, all 234 BLCA patients were stratified by the age (65 years old) into a younger dataset $(n=86)$ and an elder dataset $(n$ $=148$ ). The four-lncRNA signature could classify the younger dataset into a high-risk group $(n=45)$ and a lowrisk group $(n=41)$ with significantly different survival (log-rank test $p$-value $=1.64 \mathrm{E}-04$, median 6.00 months vs. 6.77 months; Figure 3A). Similarly, the four-lncRNA signature was also able to classify the elder dataset into a high-risk group $(n=76)$ and a low-risk group $(n=72)$ with significantly different survival (log-rank test $p$-value $=1.02 \mathrm{E}-04$, median 6.45 months vs. 7.60 months; Figure 3B). Then all patients were further stratified by the tumor stage into an early dataset (stage II and stage III, $n=156$ ) and a late dataset (stage IV, $n=78$ ). Similar prognostic power of the four-lncRNA signature was significant in both the early dataset and late dataset. Patients in the early dataset were classified into a high-risk group $(n=$ $80)$ with shorter survival and a low-risk group $(n=76)$ with longer survival (log-rank test $p$-value $=2.25 \mathrm{E}-04$, median 6.48 months $v s .7 .05$ months; Figure 3C). Similar results were observed in the late dataset (log-rank test $p$ $=1.55 \mathrm{E}-05$, median 5.93 months vs. 7.73 months; Figure 3D). Finally, all patients were stratified by the subtype into a non-papillary dataset $(n=165)$ and a papillary dataset $(n=69)$. Significant differences in patients' survival between the high-risk groups and the low-risk groups were also observed in the two datasets (log-rank test $p$-value $=5.52 \mathrm{E}-05$, median 6.50 months vs. 7.13 months, Figure $3 \mathrm{E}$; log-rank test $p$-value $=4.33 \mathrm{E}-04$, median 3.73 months vs. 7.47 months, Figure 3F). The results of multivariate Cox regression analyses, together with the stratified analyses, demonstrated that the prognostic power of the four-lncRNA signature was independent of other clinical variables for survival prediction of patients with BLCA.

\section{Functional characteristics of the four prognostic IncRNAs}

To investigate potential functional roles of the four prognostic IncRNAs in BLCA tumorigenesis, we carried out functional enrichment analyses to predict their functions [44]. We first calculate Spearman correlation coefficients between lncRNAs and protein-coding genes by examining the paired lncRNA and the protein-coding gene expression profiles of 234 patients with BLCA. A total of 1405 protein-coding genes were significantly correlated with at least one of four prognostic lncRNAs (Spearman correlation coefficient $>0.40$ ). Functional enrichment analyses of GO and KEGG pathways revealed that 1405 protein-coding genes were significantly enriched in $50 \mathrm{GO}$ terms (a p-value of $<0.05$ and an enrichment score of $>1.0$ ) and 7 KEGG pathways (a $p$-value of $<0.05$ and a fold enrichment of $>2.0$ ). These functionally related 
GO terms were mainly organized into seven functional clusters including assembly and disassembly of protein and macromolecules, transcription, signal transduction and response to stimulus, cell apoptosis and death, metabolic and catabolic process, cell development, carbohydrate metabolic (Figure 4A). Seven significantly enriched KEGG pathways were observed including tight junction, aldosterone-regulated sodium reabsorption, pathogenic escherichia coli infection, adherens junction, valine, leucine and isoleucine degradation, p53 signaling pathway, glycosphingolipid biosynthesis (Figure 4B). These results of the functional enrichment analyses suggested that the four prognostic lncRNAs may participate in tumorigenesis through regulating or interacting protein-coding genes to affect known BLCA-related biological processes and pathways.

\section{DISCUSSION}

Considerable efforts have been made during the past years to identify expression-based prognostic biomarkers for bladder cancer at protein-coding genes and miRNAs levels [45-47]. More recently, accumulated evidence indicates that dysregulated lncRNAs are implicated in various tumorigenesis processes including proliferation, invasion and apoptosis by acting as tumor oncogenes or suppressor, which has developed a new area for biomarkers. Additionally, plenty of aberrant lncRNA expression in multiple cancers was discovered by the transcriptional profiling analyses [48, 49], highlighting their potential roles as novel independent biomarkers for cancer prognosis. By now, several expression-based lncRNA signatures have been identified in glioblastoma, oesophageal squamous cell carcinoma, breast cancer, colorectal cancer, non-small cell lung cancer, multiple myeloma and ovarian cancer. Compared with proteincoding genes, lncRNAs exhibit greater tissue-, diseaseand developmental stage-specific expression, and their expression is more closely related to the tumor status and biological functions [50-54]. Indeed, several lncRNAs, such as HOTAIR, SPRY4-IT1, SUMOIP3 and PANDAR have been found to be associated with bladder prognosis $[40,43]$. However, to date, the prognostic power of expression-based lncRNA signature for predicting survival in patients with BLCA has not yet been investigated.

Here, we performed a comprehensive analysis for lncRNA expression profiles and corresponding clinical information of BLCA patients in the training dataset, and identified four lncRNAs significantly associated with patients' survival and constructed a four-lncRNA signature that can effectively predict survival of BLCA patients. Further ROC curve analysis demonstrated the competitive performance of the four-lncRNA signature for predicting 5-year survival in the training dataset. Then the prognostic power of the four-lncRNA signature was validated in an independent non-overlapping dataset and an entire dataset, demonstrated good reliability and reproducibility of the four-lncRNA signature for predicting BLCA patients' survival. Next, we performed multivariate Cox regression analyses in each dataset
A

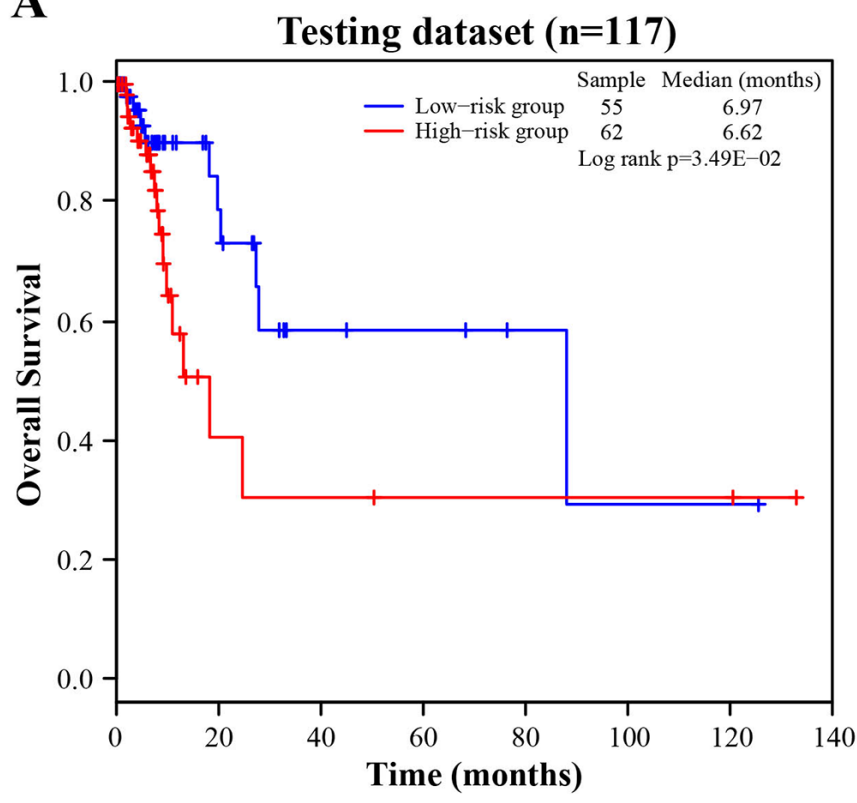

B

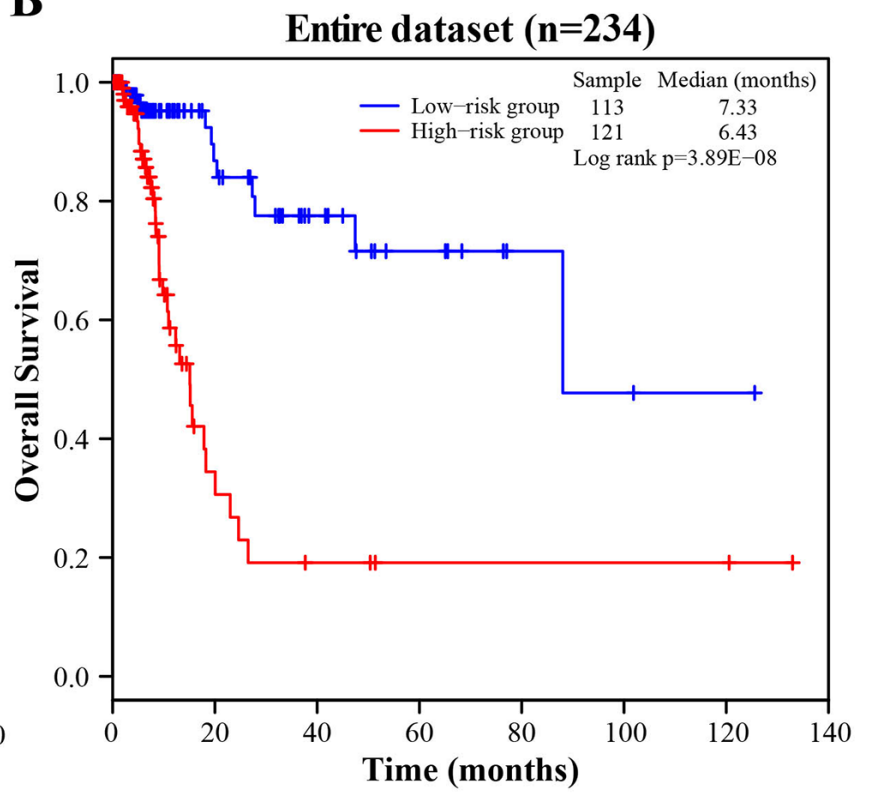

Figure 2: The Kaplan-Meier curves of overall survival between high-risk and low-risk patients in the testing and entire dataset. A. The Kaplan-Meier curves for the testing dataset. B. The Kaplan-Meier curves for the entire dataset. 
A

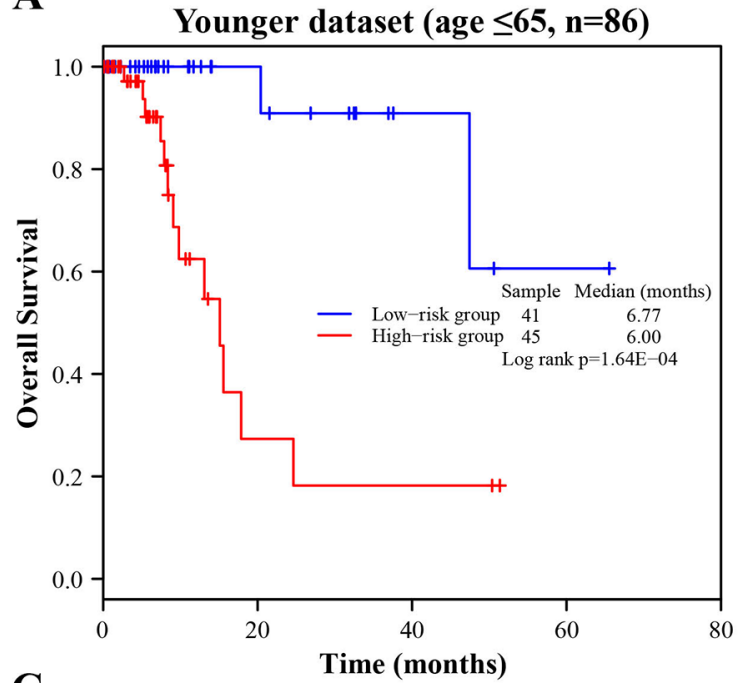

C

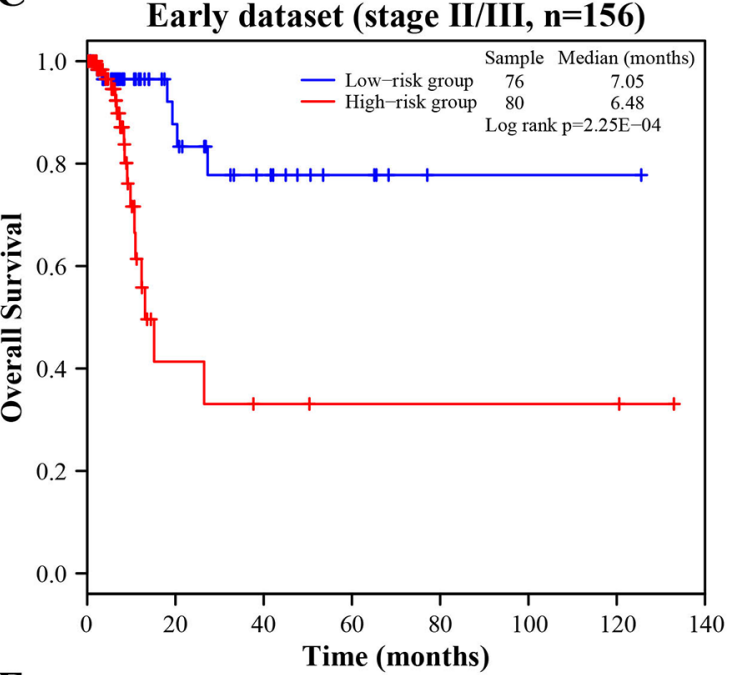

$\mathbf{E}$

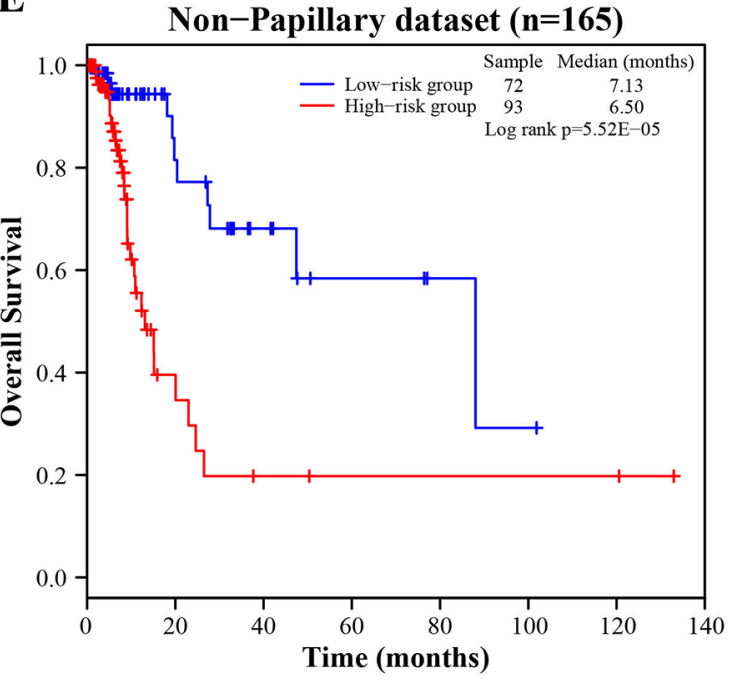

B

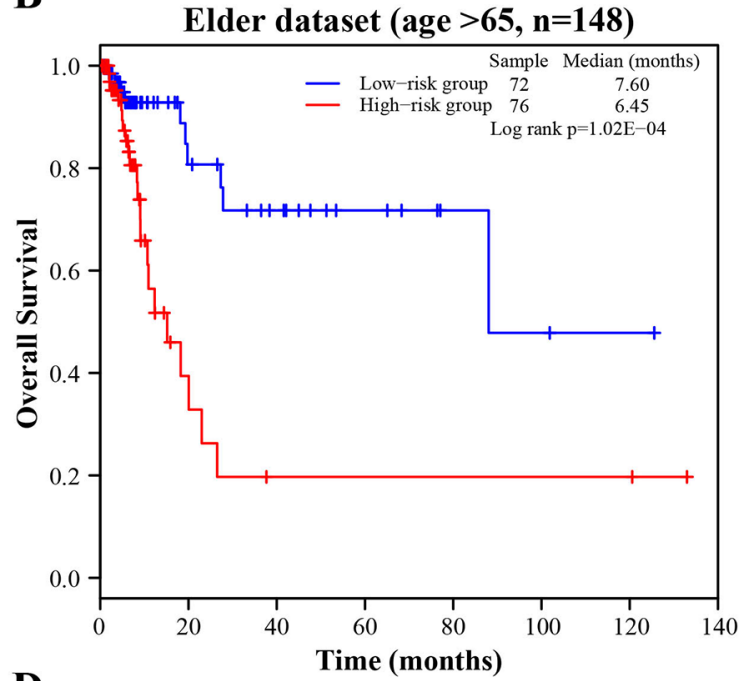

D

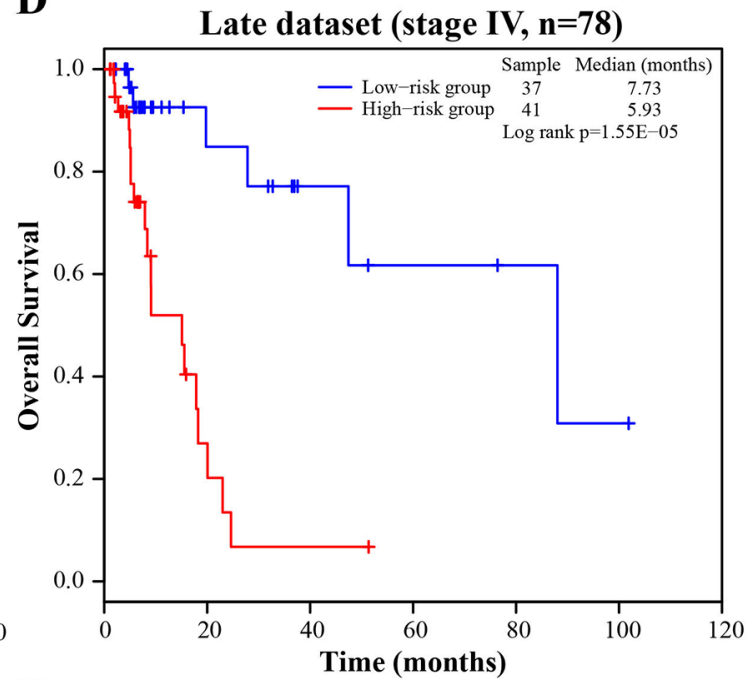

$\mathbf{F}$

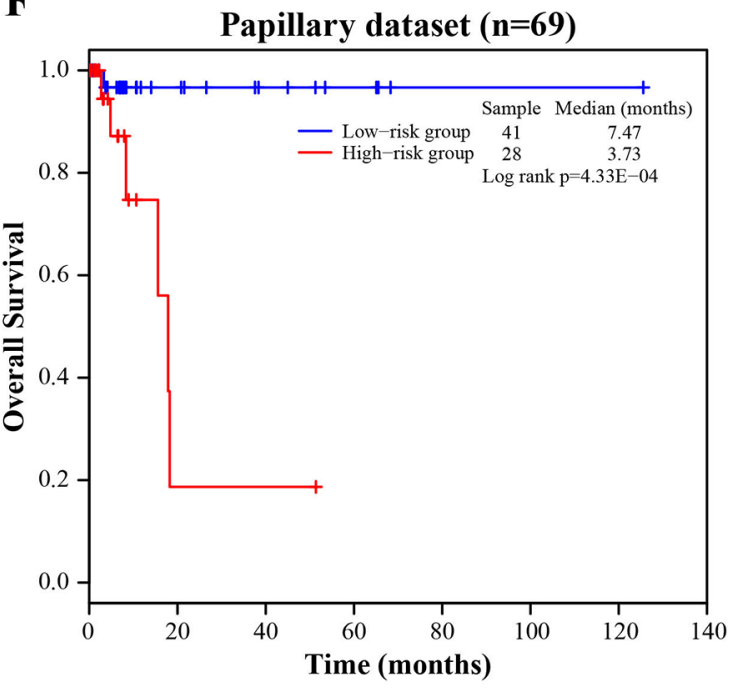

Figure 3. Survival analyses of all BLCA patients stratified by age, stage, tumor subtype with the four-IncRNA signature. A. The Kaplan-Meier curves for the younger dataset (age $\leq 65, \mathrm{n}=86$ ). B. The Kaplan-Meier curves for the elder dataset (age $>65$, $\mathrm{n}=148$ ). C. The Kaplan-Meier curves for the early dataset (stage II/III, $\mathrm{n}=156$ ). D. The Kaplan-Meier curves for the late dataset (stage IV, $n=78$ ). E. The Kaplan-Meier curves for the non-papillary dataset (subtype of non-papillary, $n=165$ ). F. The Kaplan-Meier curves for papillary dataset (subtype of papillary, $\mathrm{n}=69$ ). 
to evaluate whether the prognostic power of the fourlncRNA signature was independently of other clinical variables, including age, gender, subtype and tumor stage. The four-lncRNA signature was demonstrated to still maintain an independent association with patients' survival after adjustment for other clinical variables. In the stratified analyses, the four-lncRNA signature showed prognostic power for the younger dataset (age $\leq 65)$ and the elder dataset (age $>65$ ), in which patients belonging to the two datasets could be classified into high-risk and low-risk groups with significant differences in patients' survival. Similar prognostic power of the four-lncRNA signature was also significant in the early dataset (stage II and stage III) and the late dataset (stage IV), in which patients in the two datasets were able to classified into high-risk and low-risk groups with significantly different survival. Moreover, similar results were observed in the non-papillary dataset and the papillary dataset. Taken

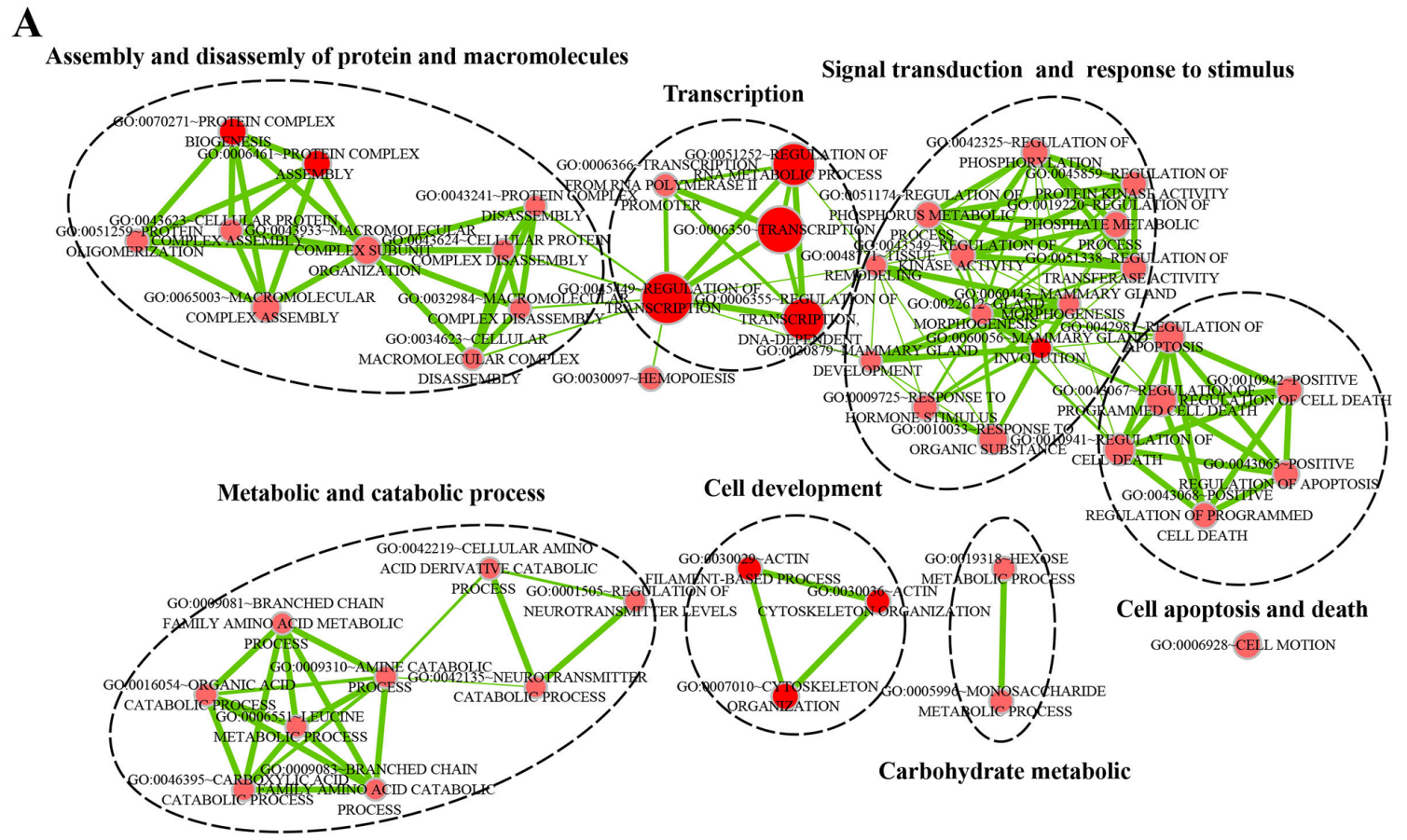

B

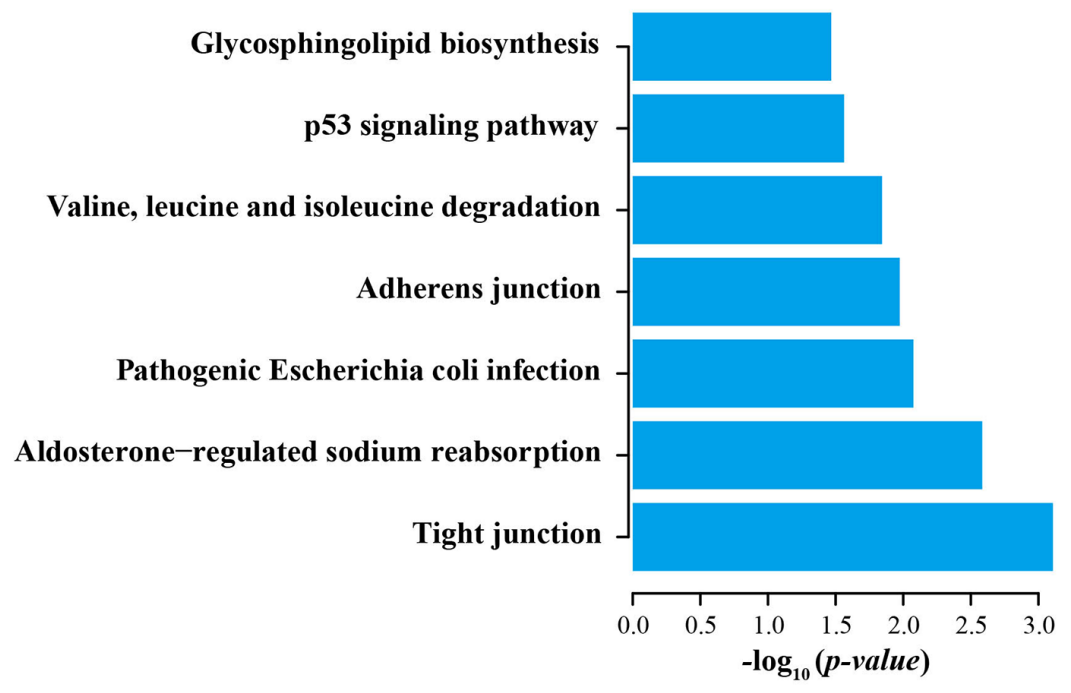

Figure 4: Functional enrichment analyses of the protein-coding genes co-expressed with the four prognostic IncRNAs. A. The functional enrichment map of GO terms. Each node represents a GO term. An edge represents the overlap of the shared genes between connecting terms. Node size represents the number of gene in the GO terms. Color intensity is proportional to enrichment significance. B. Significantly enriched KEGG pathway. 
Table 3: Clinical characteristics of patients with BLCA in this study

\begin{tabular}{|c|c|c|c|}
\hline Characteristics & $\begin{array}{c}\text { Training dataset } \\
(\mathrm{n}=117)\end{array}$ & $\begin{array}{c}\text { Testing dataset } \\
(\mathrm{n}=117)\end{array}$ & $\begin{array}{c}\text { Entire dataset } \\
(\mathrm{n}=\mathbf{2 3 4})\end{array}$ \\
\hline \multicolumn{4}{|l|}{ Vital status } \\
\hline Alive & $96(82.1 \%)$ & $91(77.8 \%)$ & 187 (79.9\%) \\
\hline Dead & $21(17.9 \%)$ & $26(22.2 \%)$ & $47(20.1 \%)$ \\
\hline \multicolumn{4}{|l|}{ Age } \\
\hline$\leq 65$ & $50(42.7 \%)$ & $36(30.8 \%)$ & $86(36.8 \%)$ \\
\hline$>65$ & $67(57.3 \%)$ & $81(69.2 \%)$ & $148(63.2 \%)$ \\
\hline \multicolumn{4}{|l|}{ Gender } \\
\hline Female & $31(26.5 \%)$ & $28(23.9 \%)$ & $59(25.2 \%)$ \\
\hline Male & $86(73.5 \%)$ & $89(76.1 \%)$ & $175(74.8 \%)$ \\
\hline \multicolumn{4}{|l|}{ Subtype } \\
\hline Non-papillary & $82(70.1 \%)$ & $83(70.9 \%)$ & $165(70.5 \%)$ \\
\hline Papillary & $35(29.9 \%)$ & $34(29.1 \%)$ & $69(29.5 \%)$ \\
\hline \multicolumn{4}{|l|}{ Stage } \\
\hline II & $41(35.0 \%)$ & $29(24.8 \%)$ & $70(29.9 \%)$ \\
\hline III & $37(31.6 \%)$ & $49(41.9 \%)$ & $86(36.8 \%)$ \\
\hline IV & $39(33.3 \%)$ & $39(33.3 \%)$ & $78(33.3 \%)$ \\
\hline
\end{tabular}

together, these results demonstrated that the prognostic power of the four-lncRNA signature was independent of other clinical variables for survival prediction of patients with BLCA.

Up to date, although more than tens of thousands of IncRNAs have been discovered in humans over the past few decades [55], only a handful of lncRNAs were functionally well-characterized and the functional study of $\operatorname{lncRNAs}$ remains in its infancy. Previous studies have suggested that lncRNAs participated in biological processes and pathways by regulating or interacting with proteincoding genes involved in the same processes, making it possible to infer lncRNA biological functions from their co-expressed protein-coding genes [44, 56, 57]. To detect the biological implication of the four prognostic lncRNAs in BLCA, we performed GO and KEGG functional enrichment analyses for co-expressed protein-coding genes. The results indicated the important functional roles of the four prognostic lncRNAs in tumorigenesis.

In summary, by performing a comprehensive analysis for lncRNA expression profiles and corresponding clinical information, our study identified four prognostic IncRNAs were significantly associated with BLCA patients' survival and constructed a four-lncRNA signature that can effectively predict patients' survival. The prognostic power of the four-lncRNA signature was independent of other clinical variables, and showed superior performance compared to known traditional clinical variables in a way. Our results demonstrated the
four-lncRNA signature can function as novel independent biomarkers for BLCA prognosis and provided novel insights into understanding the underlying molecular mechanism of BLCA.

\section{MATERIALS AND METHODS}

\section{BLCA datasets and patient information}

The IncRNA expression profiles and corresponding clinical information of BLCA patients were obtained from The Cancer Genome Atlas (TCGA) data portal (up to May 27, 2016; https://gdc-portal.nci.nih.gov/). A total of 234 patients were enrolled in this study after removal of patients without available clinical information. Clinical information of BLCA patients used in this study, including age, gender, subtype and stage. More detailed clinical characteristics of all 234 BLCA patients in this study were listed in Table 3.

\section{Acquisition of IncRNA expression profiles}

The lncRNAs derived from TCGA and lncRNAs from GENCODE project [58] were cross-reference by Ensemble ID to reduce redundant. Then the lncRNA expression profiles were defined as those with an average $R P K M \geq 0.3$ across all 234 BLCA patients. Finally, we obtained expression profiles of 12730 lncRNAs in 234 BLCA patients. 


\section{Construction of a prognostic IncRNA signature}

The IncRNAs expression profiles were normalized by $\log 2$ transformed. The association between the expression profiles of each IncRNA and patients' survival was evaluated in the training dataset using a univariate Cox regression analysis with survival as the dependent variable. LncRNAs whose expression profiles were significantly associated with patients' survival were identified ( $p$-value $<0.001$ ) as prognostic lncRNAs, and then subjected to a multivariate Cox regression analysis in the training dataset with survival as the dependent variable and other clinical information as covariables. Subsequently, a prognostic lncRNA signature was constructed based on a linear combination of the expression profiles of prognostic lncRNAs with weighted by the estimated regression coefficient as follows:

$$
\text { Risk Score }(R S)=\sum_{i=1}^{N}\left(\operatorname{Exp}_{i} * \mathrm{Coe}_{i}\right)
$$

where $N$ is the number of prognostic lncRNAs, $\operatorname{Exp}_{i}$ is the expression profiles of $\operatorname{lncRNA} A_{i}$, and $\mathrm{Coe}_{i}$ is the estimated regression coefficient of $\operatorname{lnc} R N A_{i}$ derived from the multivariate Cox regression analysis. The lncRNA signature could calculate a risk score for each patient. With the IncRNA signature, BLCA patients in each dataset were classified into high-risk and low-risk groups using the median risk score derived from the training dataset as a cutoff point.

\section{Statistical analysis}

Differences in patients' survival between the high-risk group and the low-risk group in each dataset were accessed by the Kaplan-Meier survival analyses, and compared by the two-sided log-rank test using the R package "survival" [59]. Furthermore, in order to evaluate whether the prognostic power of the four-lncRNA signature was independent of other clinical variables including age, gender, subtype and tumor stage, multivariate Cox regression and stratified analyses were carried out in each dataset with survival as the dependent variable, IncRNA risk score and other clinical variables as explanatory variables. Hazard ratios (HR) and 95\% confidence intervals (CI) were calculated. The time-dependent receiver operating characteristic (ROC) curve analyses within five years as the defining points were performed using the R package "survivalROCR" [60], which has been widely used to evaluate the prognostic performance for survival prediction [61]. Area under the ROC curve (AUC) values were calculated from the ROC curves. All analyses were performed using R software and Bioconductor (version 3.3.0).

\section{Functional enrichment analyses}

Spearman correlation coefficients were computed to evaluate co-expression relationships between prognostic lncRNAs and protein-coding genes. Functional enrichment analyses for the co-expressed protein-coding genes were carried out using the DAVID Bioinformatics Tool (version 6.7) [62, 63] limited to Gene ontology (GO) terms in the "Biological Process" (GOTERM-BP-FAT) and Kyoto encyclopedia of genes and genomes (KEGG) pathway categories with the human whole genome as the background. GO terms with a $p$-value of $<0.05$ and an enrichment score of $>1.0$, KEGG pathway with a $p$-value of $<0.05$ and a fold enrichment of $>2.0$ were considered as significantly enriched function annotations. Significant enrichment results were visualized and clustered based on the similar function using the Enrichment Map plugin [64] in Cytoscape (version 3.4.0) [65] and R package "goProfiles" [66].

\section{ACKNOWLEDGMENTS}

This work is supported by National Natural Science of China to Dong Dong (Grant No. is 31200956).

\section{CONFLICTS OF INTEREST}

The authors declare that they have no competing interest.

\section{Authors' contributions}

DD conceived and designed the experiments. ZB, ZW and DD analyzed the data and wrote the manuscript. All authors read and approved the final manuscript.

\section{REFERENCES}

1. Witjes JA, Comperat E, Cowan NC, De Santis M, Gakis G, Lebret T, Ribal MJ, Van der Heijden AG, Sherif A and European Association of U. EAU guidelines on muscleinvasive and metastatic bladder cancer: summary of the 2013 guidelines. Eur Urol. 2014; 65:778-792.

2. Siegel RL, Miller KD and Jemal A. Cancer statistics, 2016. CA Cancer J Clin. 2016; 66:7-30.

3. Kirkali Z, Chan T, Manoharan M, Algaba F, Busch C, Cheng L, Kiemeney L, Kriegmair M, Montironi R, Murphy WM, Sesterhenn IA, Tachibana M and Weider J. Bladder cancer: epidemiology, staging and grading, and diagnosis. Urology. 2005; 66:4-34.

4. Marta GN, Hanna SA, Gadia R, Correa SF, Silva JL and Carvalho Hde A. The role of radiotherapy in urinary bladder cancer: current status. Int Braz J Urol. 2012; 38:144-153; discussion 153-144.

5. Racioppi M, D’Agostino D, Totaro A, Pinto F, Sacco E, D’Addessi A, Marangi F, Palermo G and Bassi PF. Value of current chemotherapy and surgery in advanced and metastatic bladder cancer. Urol Int. 2012; 88:249-258. 
6. Chen J, Wang L, Tang Y, Gong G, Liu L, Chen M, Chen Z, Cui Y, Li C, Cheng X, Qi L and Zu X. Maspin enhances cisplatin chemosensitivity in bladder cancer T24 and 5637 cells and correlates with prognosis of muscleinvasive bladder cancer patients receiving cisplatin based neoadjuvant chemotherapy. J Exp Clin Cancer Res. 2016; 35:2.

7. Mercer TR, Dinger ME and Mattick JS. Long non-coding RNAs: insights into functions. Nat Rev Genet. 2009; 10:155-159.

8. Ponting CP, Oliver PL and Reik W. Evolution and functions of long noncoding RNAs. Cell. 2009; 136:629-641.

9. Kornienko AE, Guenzl PM, Barlow DP and Pauler FM. Gene regulation by the act of long non-coding RNA transcription. BMC Biol. 2013; 11:59.

10. Fatica A and Bozzoni I. Long non-coding RNAs: new players in cell differentiation and development. Nat Rev Genet. 2014; 15:7-21.

11. Geisler S and Coller J. RNA in unexpected places: long non-coding RNA functions in diverse cellular contexts. Nat Rev Mol Cell Biol. 2013; 14:699-712.

12. Tsai MC, Spitale RC and Chang HY. Long intergenic noncoding RNAs: new links in cancer progression. Cancer Res. 2011; 71:3-7.

13. Uchida $\mathrm{S}$ and Dimmeler S. Long noncoding RNAs in cardiovascular diseases. Circ Res. 2015; 116:737-750.

14. Kumar V, Westra HJ, Karjalainen J, Zhernakova DV, Esko T, Hrdlickova B, Almeida R, Zhernakova A, Reinmaa E, Vosa U, Hofker MH, Fehrmann RS, Fu J, Withoff S, Metspalu A, Franke L, et al. Human disease-associated genetic variation impacts large intergenic non-coding RNA expression. PLoS Genet. 2013; 9:e1003201.

15. Gupta RA, Shah N, Wang KC, Kim J, Horlings HM, Wong DJ, Tsai MC, Hung T, Argani P, Rinn JL, Wang Y, Brzoska P, Kong B, Li R, West RB, van de Vijver $\mathrm{MJ}$, et al. Long non-coding RNA HOTAIR reprograms chromatin state to promote cancer metastasis. Nature. 2010; 464:1071-1076.

16. Geng YJ, Xie SL, Li Q, Ma J and Wang GY. Large intervening non-coding RNA HOTAIR is associated with hepatocellular carcinoma progression. J Int Med Res. 2011; 39:2119-2128.

17. Ishibashi M, Kogo R, Shibata K, Sawada G, Takahashi Y, Kurashige J, Akiyoshi S, Sasaki S, Iwaya T, Sudo T, Sugimachi K, Mimori K, Wakabayashi G and Mori M. Clinical significance of the expression of long non-coding RNA HOTAIR in primary hepatocellular carcinoma. Oncol Rep. 2013; 29:946-950.

18. Kogo R, Shimamura T, Mimori K, Kawahara K, Imoto S, Sudo T, Tanaka F, Shibata K, Suzuki A, Komune S, Miyano $\mathrm{S}$ and Mori M. Long noncoding RNA HOTAIR regulates polycomb-dependent chromatin modification and is associated with poor prognosis in colorectal cancers. Cancer Res. 2011; 71:6320-6326.
19. Niinuma T, Suzuki H, Nojima M, Nosho K, Yamamoto H, Takamaru H, Yamamoto E, Maruyama R, Nobuoka T, Miyazaki Y, Nishida T, Bamba T, Kanda T, Ajioka Y, Taguchi T, Okahara S, et al. Upregulation of miR-196a and HOTAIR drive malignant character in gastrointestinal stromal tumors. Cancer Res. 2012; 72:1126-1136.

20. Tsai MC, Manor O, Wan Y, Mosammaparast N, Wang JK, Lan F, Shi Y, Segal E and Chang HY. Long noncoding RNA as modular scaffold of histone modification complexes. Science. 2010; 329:689-693.

21. Muller-Tidow C, Diederichs S, Thomas M and Serve H. Genome-wide screening for prognosis-predicting genes in early-stage non-small-cell lung cancer. Lung Cancer. 2004; 45 Suppl 2:S145-150.

22. Schmidt LH, Spieker T, Koschmieder S, Schaffers S, Humberg J, Jungen D, Bulk E, Hascher A, Wittmer D, Marra A, Hillejan L, Wiebe K, Berdel WE, Wiewrodt R and Muller-Tidow $\mathrm{C}$. The long noncoding MALAT-1 RNA indicates a poor prognosis in non-small cell lung cancer and induces migration and tumor growth. J Thorac Oncol. 2011; 6:1984-1992.

23. Lai MC, Yang Z, Zhou L, Zhu QQ, Xie HY, Zhang F, Wu LM, Chen LM and Zheng SS. Long non-coding RNA MALAT-1 overexpression predicts tumor recurrence of hepatocellular carcinoma after liver transplantation. Med Oncol. 2012; 29:1810-1816.

24. Gutschner T, Hammerle M and Diederichs S. MALAT1 -a paradigm for long noncoding RNA function in cancer. $\mathrm{J}$ Mol Med (Berl). 2013; 91:791-801.

25. Ellis BC, Molloy PL and Graham LD. CRNDE: A Long Non-Coding RNA Involved in CanceR, Neurobiology, and DEvelopment. Front Genet. 2012; 3:270.

26. Mourtada-Maarabouni M, Pickard MR, Hedge VL, Farzaneh F and Williams GT. GAS5, a non-protein-coding RNA, controls apoptosis and is downregulated in breast cancer. Oncogene. 2009; 28:195-208.

27. Zhou Y, Zhang X and Klibanski A. MEG3 noncoding RNA: a tumor suppressor. J Mol Endocrinol. 2012; 48:R45-53.

28. Zhang X, Gejman R, Mahta A, Zhong Y, Rice KA, Zhou Y, Cheunsuchon P, Louis DN and Klibanski A. Maternally expressed gene 3, an imprinted noncoding RNA gene, is associated with meningioma pathogenesis and progression. Cancer Res. 2010; 70:2350-2358.

29. Wang P, Ren Z and Sun P. Overexpression of the long non-coding RNA MEG3 impairs in vitro glioma cell proliferation. J Cell Biochem. 2012; 113:1868-1874.

30. Braconi C, Kogure T, Valeri N, Huang N, Nuovo G, Costinean S, Negrini M, Miotto E, Croce CM and Patel T. microRNA-29 can regulate expression of the long non-coding RNA gene MEG3 in hepatocellular cancer. Oncogene. 2011; 30:4750-4756.

31. Benetatos L, Hatzimichael E, Dasoula A, Dranitsaris G, Tsiara S, Syrrou M, Georgiou I and Bourantas KL. CpG 
methylation analysis of the MEG3 and SNRPN imprinted genes in acute myeloid leukemia and myelodysplastic syndromes. Leuk Res. 2010; 34:148-153.

32. Huarte M, Guttman M, Feldser D, Garber M, Koziol MJ, Kenzelmann-Broz D, Khalil AM, Zuk O, Amit I, Rabani M, Attardi LD, Regev A, Lander ES, Jacks T and Rinn JL. A large intergenic noncoding RNA induced by p53 mediates global gene repression in the p53 response. Cell. 2010; 142:409-419.

33. Zhang XQ, Sun S, Lam KF, Kiang KM, Pu JK, Ho AS, Lui WM, Fung CF, Wong TS and Leung GK. A long noncoding RNA signature in glioblastoma multiforme predicts survival. Neurobiol Dis. 2013; 58:123-131.

34. Li J, Chen Z, Tian L, Zhou C, He MY, Gao Y, Wang S, Zhou F, Shi S, Feng X, Sun N, Liu Z, Skogerboe G, Dong J, Yao R, Zhao Y, et al. LncRNA profile study reveals a threelncRNA signature associated with the survival of patients with oesophageal squamous cell carcinoma. Gut. 2014; 63:1700-1710.

35. Meng J, Li P, Zhang Q, Yang Z and Fu S. A four-long noncoding RNA signature in predicting breast cancer survival. J Exp Clin Cancer Res. 2014; 33:84.

36. Hu Y, Chen HY, Yu CY, Xu J, Wang JL, Qian J, Zhang X and Fang JY. A long non-coding RNA signature to improve prognosis prediction of colorectal cancer. Oncotarget. 2014; 5:2230-2242. doi: 10.18632/oncotarget.1895.

37. Zhou M, Guo M, He D, Wang X, Cui Y, Yang H, Hao D and Sun J. A potential signature of eight long non-coding RNAs predicts survival in patients with non-small cell lung cancer. J Transl Med. 2015; 13:231.

38. Zhou M, Zhao H, Wang Z, Cheng L, Yang L, Shi H, Yang $\mathrm{H}$ and Sun J. Identification and validation of potential prognostic lncRNA biomarkers for predicting survival in patients with multiple myeloma. J Exp Clin Cancer Res. 2015; 34:102.

39. Zhou M, Sun Y, Sun Y, Xu W, Zhang Z, Zhao H, Zhong Z and Sun J. Comprehensive analysis of lncRNA expression profiles reveals a novel lncRNA signature to discriminate nonequivalent outcomes in patients with ovarian cancer. Oncotarget. 2016; 7:32433-48. doi: 10.18632/oncotarget.8653.

40. Yan TH, Lu SW, Huang YQ, Que GB, Chen JH, Chen YP, Zhang HB, Liang XL and Jiang JH. Upregulation of the long noncoding RNA HOTAIR predicts recurrence in stage $\mathrm{Ta} / \mathrm{T} 1$ bladder cancer. Tumour Biol. 2014; 35:10249-10257.

41. Zhao XL, Zhao ZH, Xu WC, Hou JQ and Du XY. Increased expression of SPRY4-IT1 predicts poor prognosis and promotes tumor growth and metastasis in bladder cancer. Int J Clin Exp Pathol. 2015; 8:1954-1960.

42. Zhan Y, Liu Y, Wang C, Lin J, Chen M, Chen X, Zhuang C, Liu L, Xu W, Zhou Q, Sun X, Zhang Q, Zhao G and Huang W. Increased expression of SUMO1P3 predicts poor prognosis and promotes tumor growth and metastasis in bladder cancer. Oncotarget. 2016; 7:16038-16048. doi: 10.18632/oncotarget.6946.
43. Zhan Y, Lin J, Liu Y, Chen M, Chen X, Zhuang C, Liu L, Xu W, Chen Z, He A, Zhang Q, Sun X, Zhao G and Huang W. Up-regulation of long non-coding RNA PANDAR is associated with poor prognosis and promotes tumorigenesis in bladder cancer. J Exp Clin Cancer Res. 2016; 35:83.

44. Liao Q, Liu C, Yuan X, Kang S, Miao R, Xiao H, Zhao G, Luo H, Bu D, Zhao H, Skogerbo G, Wu Z and Zhao Y. Large-scale prediction of long non-coding RNA functions in a coding-non-coding gene co-expression network. Nucleic Acids Res. 2011; 39:3864-3878.

45. Jeong P, Ha YS, Cho IC, Yun SJ, Yoo ES, Kim IY, Choi YH, Moon SK and Kim WJ. Three-gene signature predicts disease progression of non-muscle invasive bladder cancer. Oncol Lett. 2011; 2:679-684.

46. Kim WJ, Kim SK, Jeong P, Yun SJ, Cho IC, Kim IY, Moon SK, Um HD and Choi YH. A four-gene signature predicts disease progression in muscle invasive bladder cancer. Mol Med. 2011; 17:478-485.

47. Zhou H, Tang K, Xiao H, Zeng J, Guan W, Guo X, Xu H and Ye Z. A panel of eight-miRNA signature as a potential biomarker for predicting survival in bladder cancer. J Exp Clin Cancer Res. 2015; 34:53.

48. Qiu MT, Hu JW, Yin R and Xu L. Long noncoding RNA: an emerging paradigm of cancer research. Tumour Biol. 2013; 34:613-620.

49. Gibb EA, Vucic EA, Enfield KS, Stewart GL, Lonergan KM, Kennett JY, Becker-Santos DD, MacAulay CE, Lam S, Brown CJ and Lam WL. Human cancer long non-coding RNA transcriptomes. PLoS One. 2011; 6:e25915.

50. Zhang H, Chen Z, Wang X, Huang Z, He Z and Chen Y. Long non-coding RNA: a new player in cancer. J Hematol Oncol. 2013; 6:37.

51. Gutschner T and Diederichs S. The hallmarks of cancer: a long non-coding RNA point of view. RNA Biol. 2012; 9:703-719.

52. Cabili MN, Trapnell C, Goff L, Koziol M, Tazon-Vega B, Regev A and Rinn JL. Integrative annotation of human large intergenic noncoding RNAs reveals global properties and specific subclasses. Genes Dev. 2011; 25:1915-1927.

53. Brunner AL, Beck AH, Edris B, Sweeney RT, Zhu SX, Li R, Montgomery K, Varma S, Gilks T, Guo X, Foley JW, Witten DM, Giacomini CP, Flynn RA, Pollack JR, Tibshirani R, et al. Transcriptional profiling of long non-coding RNAs and novel transcribed regions across a diverse panel of archived human cancers. Genome Biol. 2012; 13:R75.

54. Gibb EA, Brown CJ and Lam WL. The functional role of long non-coding RNA in human carcinomas. Mol Cancer. $2011 ; 10: 38$.

55. Hangauer MJ, Vaughn IW and McManus MT. Pervasive transcription of the human genome produces thousands of previously unidentified long intergenic noncoding RNAs. PLoS Genet. 2013; 9:e1003569.

56. Guo X, Gao L, Liao Q, Xiao H, Ma X, Yang X, Luo H, Zhao G, Bu D, Jiao F, Shao Q, Chen R and Zhao Y. Long 
non-coding RNAs function annotation: a global prediction method based on bi-colored networks. Nucleic Acids Res. 2013; 41:e35.

57. Ma H, Hao Y, Dong X, Gong Q, Chen J, Zhang J and Tian W. Molecular mechanisms and function prediction of long noncoding RNA. ScientificWorldJournal. 2012; 2012:541786.

58. Harrow J, Frankish A, Gonzalez JM, Tapanari E, Diekhans M, Kokocinski F, Aken BL, Barrell D, Zadissa A, Searle S, Barnes I, Bignell A, Boychenko V, Hunt T, Kay M, Mukherjee G, et al. GENCODE: the reference human genome annotation for The ENCODE Project. Genome Res. 2012; 22:1760-1774.

59. T T. (2016). A Package for Survival Analysis in S. version 2.39-5.

60. Sing T, Sander O, Beerenwinkel N and Lengauer T. ROCR: visualizing classifier performance in R. Bioinformatics. 2005; 21:3940-3941.

61. Heagerty PJ and Zheng Y. Survival model predictive accuracy and ROC curves. Biometrics. 2005; 61:92-105.
62. Huang da W, Sherman BT and Lempicki RA. Systematic and integrative analysis of large gene lists using DAVID bioinformatics resources. Nat Protoc. 2009; 4:44-57.

63. Huang da W, Sherman BT and Lempicki RA. Bioinformatics enrichment tools: paths toward the comprehensive functional analysis of large gene lists. Nucleic Acids Res. 2009; 37:1-13.

64. Merico D, Isserlin R, Stueker O, Emili A and Bader GD. Enrichment map: a network-based method for gene-set enrichment visualization and interpretation. PLoS One. 2010; 5:e13984.

65. Shannon P, Markiel A, Ozier O, Baliga NS, Wang JT, Ramage D, Amin N, Schwikowski B and Ideker T. Cytoscape: a software environment for integrated models of biomolecular interaction networks. Genome Res. 2003; 13:2498-2504.

66. Salicru M, Ocana J and Sanchez-Pla A. Comparison of lists of genes based on functional profiles. BMC Bioinformatics. 2011; $12: 401$. 